\title{
Conserved associations between G- quadruplex-forming DNA motifs and virulence gene families in malaria parasites
}

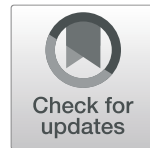

\author{
Hunter L. Gage and Catherine J. Merrick ${ }^{*}$ (D)
}

\begin{abstract}
Background: The Plasmodium genus of malaria parasites encodes several families of antigen-encoding genes. These genes tend to be hyper-variable, highly recombinogenic and variantly expressed. The best-characterized family is the var genes, exclusively found in the Laveranian subgenus of malaria parasites infecting humans and great apes. Var genes encode major virulence factors involved in immune evasion and the maintenance of chronic infections. In the human parasite P. falciparum, var gene recombination and diversification appear to be promoted by G-quadruplex (G4) DNA motifs, which are strongly associated with var genes in P. falciparum. Here, we investigated how this association might have evolved across Plasmodium species - both Laverania and also more distantly related species which lack vars but encode other, more ancient variant gene families.

Results: The association between var genes and G4-forming motifs was conserved across Laverania, spanning $~ 1$ million years of evolutionary time, with suggestive evidence for evolution of the association occurring within this subgenus. In rodent malaria species, G4-forming motifs were somewhat associated with pir genes, but this was not conserved in the Laverania, nor did we find a strong association of these motifs with any gene family in a second outgroup of avian malaria parasites. Secondly, we compared two different G4 prediction algorithms in their performance on extremely A/T-rich Plasmodium genomes, and also compared these predictions with experimental data from G4-seq, a DNA sequencing method for identifying G4-forming motifs. We found a surprising lack of concordance between the two algorithms and also between the algorithms and G4-seq data.

Conclusions: G4-forming motifs are uniquely strongly associated with Plasmodium var genes, suggesting a particular role for G4s in recombination and diversification of these genes. Secondly, in the A/T-rich genomes of Plasmodium species, the choice of prediction algorithm may be particularly influential when studying G4s in these important protozoan pathogens.
\end{abstract}

Keywords: Plasmodium, Laverania, Malaria, G quadruplex, var genes, pir genes

\section{Background}

Malaria is caused by protozoan Plasmodium parasites: in humans it causes considerable morbidity and is still responsible for almost half a million deaths each year [1]. Most severe cases of human malaria are caused by

\footnotetext{
* Correspondence: cjm48@cam.ac.uk

Department of Pathology, Cambridge University, Tennis Court Road, Cambridge CB2 1QP, UK
}

(c) The Author(s). 2020 Open Access This article is licensed under a Creative Commons Attribution 4.0 International License, which permits use, sharing, adaptation, distribution and reproduction in any medium or format, as long as you give appropriate credit to the original author(s) and the source, provide a link to the Creative Commons licence, and indicate if changes were made. The images or other third party material in this article are included in the article's Creative Commons licence, unless indicated otherwise in a credit line to the material. If material is not included in the article's Creative Commons licence and your intended use is not permitted by statutory regulation or exceeds the permitted use, you will need to obtain permission directly from the copyright holder. To view a copy of this licence, visit http://creativecommons.org/licenses/by/4.0/. The Creative Commons Public Domain Dedication waiver (http://creativecommons.org/publicdomain/zero/1.0/) applies to the data made available in this article, unless otherwise stated in a credit line to the data.

Plasmodium falciparum, but a further five parasite species can infect humans and there are many more species that infect rodents, birds and other vertebrates. In all vertebrate hosts, the disease involves cyclical infection of erythrocytes by Plasmodium parasites. The infected cells are exposed to circulating immune factors and also to splenic clearance, yet many malarias can lead to both chronic and repeated infections, indicating that the parasites have considerable capacities for immune evasion. 
These capacities have been linked, in several species, to the variant expression of virulence factors that are exposed on the infected erythrocyte surface and are encoded by highly variable families of variantly-expressed virulence genes.

Such gene families are best-characterized in $P$. falciparum, where the var gene family encodes $\sim 60$ variants of $P$. falciparum Erythrocyte Membrane Protein 1 (PfEMP1) [2-4], an adhesin which allows infected erythrocytes to adhere to the vascular endothelium and avoid splenic clearance. PfEMP1 proteins are critical virulence factors and they also contribute to disease, exacerbating vascular occlusion, hypoxia and lethal syndromes such as cerebral malaria. Var genes are restricted to the Laveranian subgenus of Plasmodium, which infects great apes and includes the human-infecting $P$. falciparum species [5], but other Plasmodium species encode other gene families that may have similar roles, including the sicavar family in the macaque parasite $P$. knowlesi $[6,7]$ and the pir family which appears widely in many species from rodent to human malarias [8, 9] (Fig. 1).

Effective antigenic variation over long periods of time requires highly regulated and mutually exclusive gene expression, and this has indeed been well characterized in the case of the var genes [12]. Other families, including sicavar [13] and pir [14], appear to have similar dynamics, albeit with less tight mutually-exclusive regulation. In the case of $P$. falciparum, var genes are regulated by epigenetic silencing and expression switching [12], and additional antigenic diversity is generated within the $\sim 60$-member gene family via frequent recombination during both mitosis and meiosis $[15,16]$. Thus, var gene regulation is a key virulence mechanism for the maintenance of chronic infections caused by P. falciparum: understanding this at the molecular level is an area of great interest in malaria biology.

A decade ago, it was first observed that many var genes are associated with DNA motifs that could form G-quadruplexes (G4s) [17]. G4s are DNA or RNA secondary structures of the general form $G_{3} N_{(0-11)} G_{3} N_{(0-}$ ${ }_{11)} G_{3} N_{(0-11)} G_{3}$. They are composed of G-quartets, planar arrangements formed from Hoogsteen hydrogen bonding between four guanines, which stack on top of one another to form four-stranded, non-helical structures. Each G-quartet is typically stabilized by a monovalent cation $\left(\mathrm{Na}^{+}\right.$or $\left.\mathrm{K}^{+}\right)$, and short loops of other nucleotides run between them. G4s can form intra- or intermolecularly, and can involve one, two or four oligonucleotide strands, but the 'simplest' intramolecular form is the best studied and its ability to fold both in vitro and in vivo has been shown in human cells [18]. G4s distort the double-helical structure of DNA, and can play important roles in the regulation of gene transcription [19], DNA replication [20] and telomere maintenance [21] in eukaryotes. Their potential role in var gene biology is of clear interest, particularly because Plasmodium genomes tend to be extremely G/C-poor [22] and accordingly have a striking paucity of G-rich G4-forming motifs. This, in turn, strongly suggests that there is a selective advantage for conserving G4 motifs that appear together with var genes.

Investigations conducted both in silico and in cultured parasites have recently established that G4-forming motifs indeed have a probable role in recombination and evolution of var genes in $P$. falciparum. The motifs strongly associate with recombination breakpoints that were observed in var genes when parasites were cultured over many mitotic cycles, evolving new gene variants [23]. Furthermore, the knockdown of a RecQ helicase homolog called PfWRN dramatically increased the rate of var gene recombination, as would be expected if this helicase was responsible for unwinding G4s and modulating their recombinogenic effects [24].

Here, we set out to examine in detail how the association between G4s and virulence genes might have evolved across the Plasmodium genus, and the extent to which the association is conserved among Plasmodium species. Our previous work had suggested that the association is not unique to $P$. falciparum: it also appeared in a second, closely-related Laveranian genome, $P$. reichenowi, and potentially also in $P$. knowlesi, which has sicavar genes rather than var genes [23]. (Analysis of $P$. knowlesi, however, was complicated by two factors - this genome is much less G/C-poor than P. falciparum, leading to a higher density of G-rich motifs overall, and it also contains, scattered throughout its chromosomes, many repeats of telomere sequence containing repeated guanine triads that are inherently G4-forming.) Finally, we had observed that in the rodent malaria species $P$. berghei, which has no var genes but does encode pir genes, G4-forming motifs were co-distributed with the pirs, albeit less strongly than was observed with vars [23].

This work raised further questions that could not be adequately answered with the genomes available at the time, but can now be addressed due to the recent publication of well-assembled, long-read sequences of the genomes of all seven known Laveranian species [5]. $P$. adleri and P. gaboni are relatively ancient ape parasites termed 'Clade A' Laverania; P. billcollinsi, P. blacklocki, $P$. reichenowi and $P$. praefalciparum are closer relatives of P. falciparum termed 'Clade B' (Fig. 1). We investigated whether the G4-var association is conserved across Laverania, and the additional possibility that G4s in some Laverania could be co-distributed with other variant gene families as well as (or instead of) vars. Notably, although the var gene family is uniquely and ubiquitously present in Laverania, its copy numbers vary by 


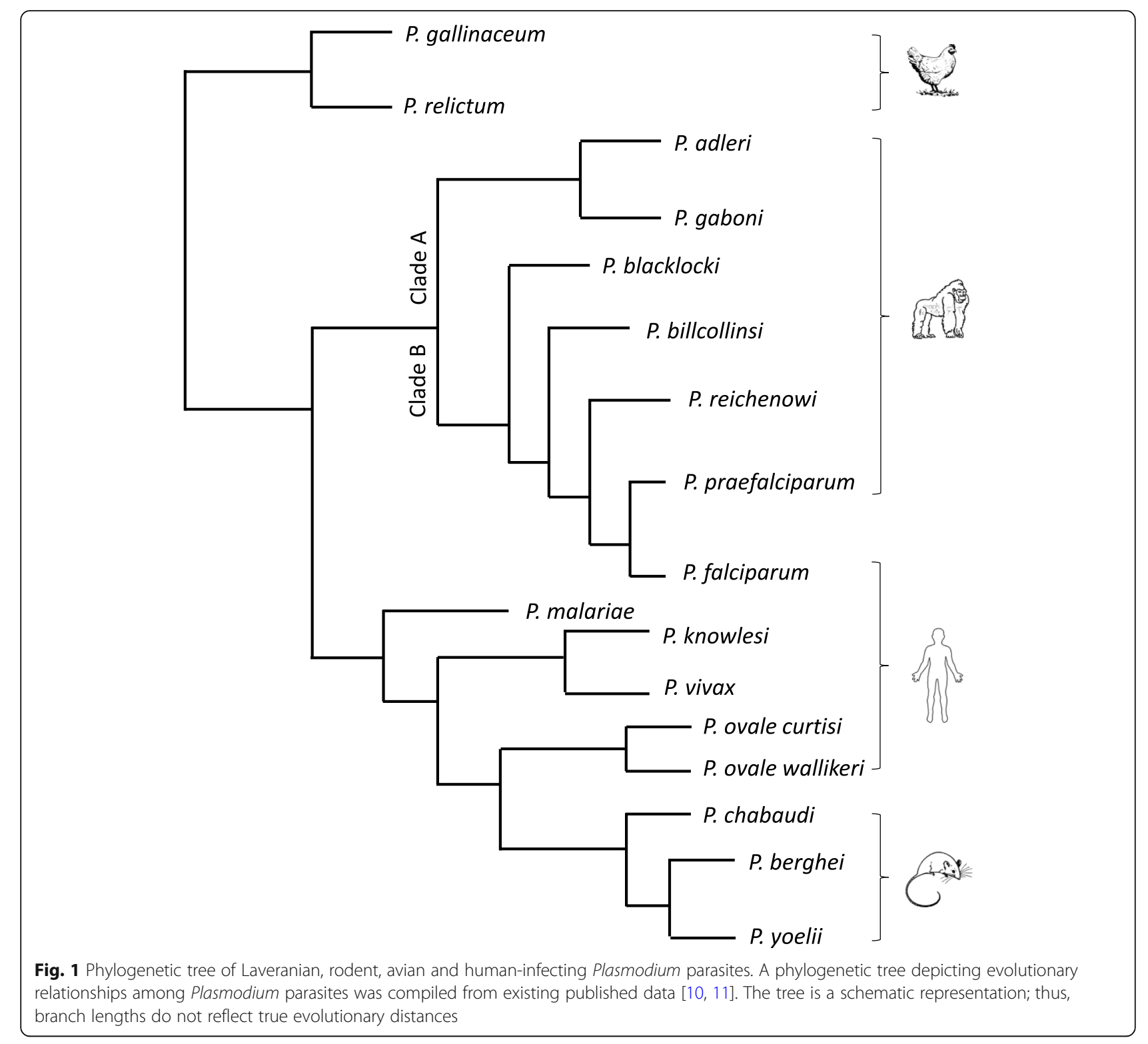

3-4 times across the subgenus, and further cladespecific expansions or contractions of gene families have also occurred: for example, Clade A Laverania have very few pirs, but encode a greatly expanded family of clags [5]. It therefore seemed possible that G4s were once associated with more ancient multigene families - as per the pirs in P. berghei - and that they might still be so in earlier Laveranian species, having only recently evolved to be exclusively co-distributed with var genes in species such as P. falciparum. If true, this would have interesting implications for G4-forming motifs as potential 'markers' of variant gene families that experience heavy selective pressure to evolve via regular recombination.

Conducting this analysis raised a second important question: how best to predict G4-forming motifs in Plasmodium genomes? Several generations of genome- scanning algorithms now exist for this purpose, but all have been primarily trained - and tested - on G/C/A/Tbalanced genomes such as the human genome. It was important therefore to establish how different predictive algorithms perform on extremely G/C-poor genomes, and also to characterize the distribution of 'canonical' versus 'non-canonical' G4s in these genomes. Canonical motifs take the stereotypical form $G_{3} N_{m} G_{3} N_{m} G_{3} N_{m} G_{3}$, while 'non-canonical' G4s can, for example, have two rather than three G-quartets, or individual guanines can be missing or interspersed with non-G bases, to give 'mismatched' or 'bulged' G4s. It is now recognized that non-canonical as well as canonical motifs can fold [25, 26], and that folding depends partly on contextual factors such as G-richness (the fraction of Gs in the sequence) and G-skewness (the degree of G/C asymmetry 
between the complementary strands) [27]. Therefore, we compared the performance of two different G4 prediction algorithms, QGRS Mapper [28] and G4 Hunter [27], in Plasmodium genomes. QGRS Mapper is a secondgeneration algorithm that searches for canonical G4forming sequences, while G4 Hunter takes into account G-richness and G-skewness to calculate a score reflecting G4-forming propensity, thus allowing the detection of non-canonical G4s. In addition to comparing the two algorithms, we compared their outputs with recentlypublished data from G4-seq performed on the P. falciparum genome [29]. In contrast to in silico algorithms, G4-seq predicts G4 motifs by sequencing DNA under conditions in which G4s can fold, resulting in characteristic sequencing errors [25]. Overall, a surprising degree of non-concordance appeared between the three approaches, in all the Plasmodium genomes that we analyzed.

\section{Results}

G-quadruplex-forming motifs in all Laveranian genomes are associated with var genes

In the $P$. falciparum genome, a search for putative quadruplex-forming sequences (PQSs) using QGRS Mapper was published in 2016, finding 80 PQSs, of which 35 were var-associated [23]. We repeated the search for PQSs in all members of the Laveranian subgenus, including $P$. falciparum, using two PQS prediction tools, QGRS Mapper and G4 Hunter (Fig. 2a). For QGRS Mapper, we used the same parameters as the previous publication (regex: $G_{3} N_{(0-11)} G_{3} N_{(0-11)} G_{3} N_{(0-11)} G_{3}$, max length: 45, min Ggroup: 3, loop size: $0-11$ ). Thus, only 'canonical' sequences were identified, with a moderately relaxed loop size of up to 11 nucleotides. For G4 Hunter, we used the following parameters: threshold: 1.7 , window size: 25 , csv data: grouped. This is considered a stringent threshold, at which the 'precision' of G4 Hunter (i.e. the proportion of genuine G4-forming sequences that are designated as such) should be similar to that of QGRS Mapper; the false positive rate is extremely low and the false negative rate is accordingly high ( $\sim 50 \%$ of 392 experimentally-confirmed G4-forming sequences were identified at this threshold) [27].

Consistent with the methodology of the 2016 study, we then sought PQSs that were either inside a var coding sequence or within $2 \mathrm{~kb}$ upstream or downstream of a var gene (and with no other nearer gene): these were defined as var-gene-associated PQSs. PQSs found in telomere repeats $(\mathrm{GGGTT}(\mathrm{C} / \mathrm{T}) \mathrm{A})$, which comprise the majority of $\mathrm{PQSs}$ in any Plasmodium genome, were treated separately and never considered var-associated.

Despite having similar genome sizes and similar G/C contents (Additional file 1: Table S1), the numbers of PQSs in the Laveranian genomes varied widely: $60 \pm 32$ with QGRS Mapper and $138 \pm 91$ with G4 Hunter (Fig. 2a, Additional file 2: Table S2). Importantly, this was not simply due to differences in how extensively telomere repeats had been assembled in the different genomes, because these repeats were excluded. On average, the Clade B Laverania had more PQSs than the Clade A species (Welch's t-test, two-tailed, QGRS Mapper: t-stat = 6.03, $p=0.0025$; G4 Hunter: $\mathrm{t}$-stat $=2.63, p=0.047$ ). Members of the subgenus differed in their proportions of var-associated PQSs (QGRS Mapper: $23 \pm 11.2 \%$, G4 Hunter: $43 \pm 14.9 \%$ ) (Fig. 2b), but these differences did not seem to map onto any particular evolutionary pattern. The differences could not be accounted for by differential G/C content within the var families, as this was similar throughout the subgenus (Additional file 1: Table S1). Despite the differences, however, all Laveranian species demonstrated a significant co-distribution of PQSs and var genes, compared to the expected distribution in a simulated genome in which var genes and PQSs occur at random (Additional file 3: Table S3). Indeed, in P. falciparum the QGRS Mapper algorithm found PQSs associated with $43 \%$ of var genes and the G4 Hunter algorithm (which tends to find more PQSs than QGRS Mapper) found PQSs in 70\% of all var genes - even when very stringently applied. This percentage would rise markedly if less stringent thresholds were applied so the great majority of var genes might reasonably be predicted to contain putative G4(s).

Overall, this confirms that the co-distribution of PQSs with var genes, previously reported only in two closely related Laveranian species separated by $\sim 0.19$ million years, $P$. falciparum and $P$. reichenowi [23], is actually conserved across all available Laveranian genomes, spanning at least 1 million years of evolutionary time [5]. Furthermore, in P. falciparum it was clear that many more PQSs were maintained in real var genes than in pseudogenes (Additional file 2: Table S2), adding further weight to the hypothesis that PQSs are positively selected in functional var genes.

\section{Some arrangements of var-associated PQSs are conserved in Laveranian genomes, but PQSs in upsB-var promoters are unique to $P$. falciparum}

We further investigated the relationship between PQSs and var genes in the Laverania by searching for conserved arrangements of PQSs in and near var genes. First, we noted that in all genomes, the majority of var-associated PQSs were within coding regions (Fig. $3 \mathrm{a}$ and b, black bars), though this proportion varied from approximately $60 \%$ in $P$. falciparum to nearly $100 \%$ in other species (QGRS Mapper data, Fig. 3a). This bias towards the coding regions of var genes was stronger than the genome-wide bias for PQSs to appear in coding regions (Fig. 3a and b, red bars). Additionally, most of the coding 

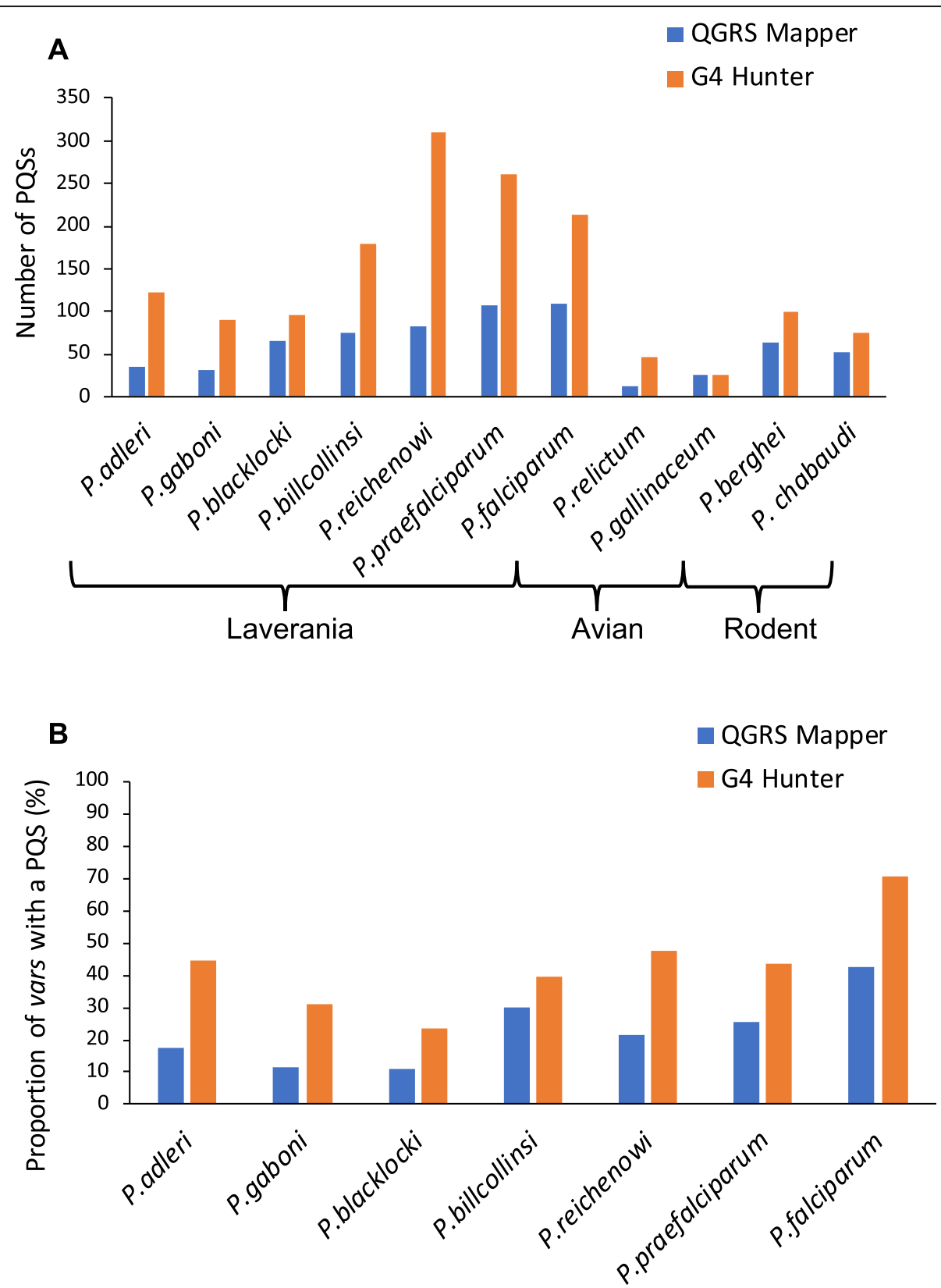

Fig. 2 Comparison of total PQSs and var-associated PQSs among Laveranian genomes. Bar plots compare the total number of PQSs in Laveranian genomes, as calculated with two different algorithms (a), and the number of var genes with at least one PQS as a percentage of the total number of var genes (b). PQSs were considered "var-associated" if they were found within a var coding sequence, or if the nearest gene, within $2 \mathrm{~kb}$, was a var gene. Copy numbers of var genes were found in PlasmoDB. Telomeric-PQSs were excluded

var-PQSs were on the antisense strand (Fig. 3c). Given that there was no apparent strand bias for PQSs overall either in the genomes as a whole, or in the non-coding UTRs of var genes - this pattern appeared to be specific to the coding location within the var gene.

Next, we analyzed the per-var distribution of PQSs, since it was clear that some var genes contained more than one PQS (this is statistically unlikely in these extremely PQS-poor genomes, and emphasizes the apparent evolutionary pressure to maintain PQSs associated with var genes). We therefore determined whether var genes were more likely to have multiple PQSs within them in some species than in others (Fig. 4). Most species tended to have one or two PQSs per var, and G4 Hunter predicted more PQSs per var than QGRS Mapper, in line with the general trend (seen in Fig. 2). Furthermore, the data from G4 Hunter suggested that the Laveranian species may be evolving towards having more 


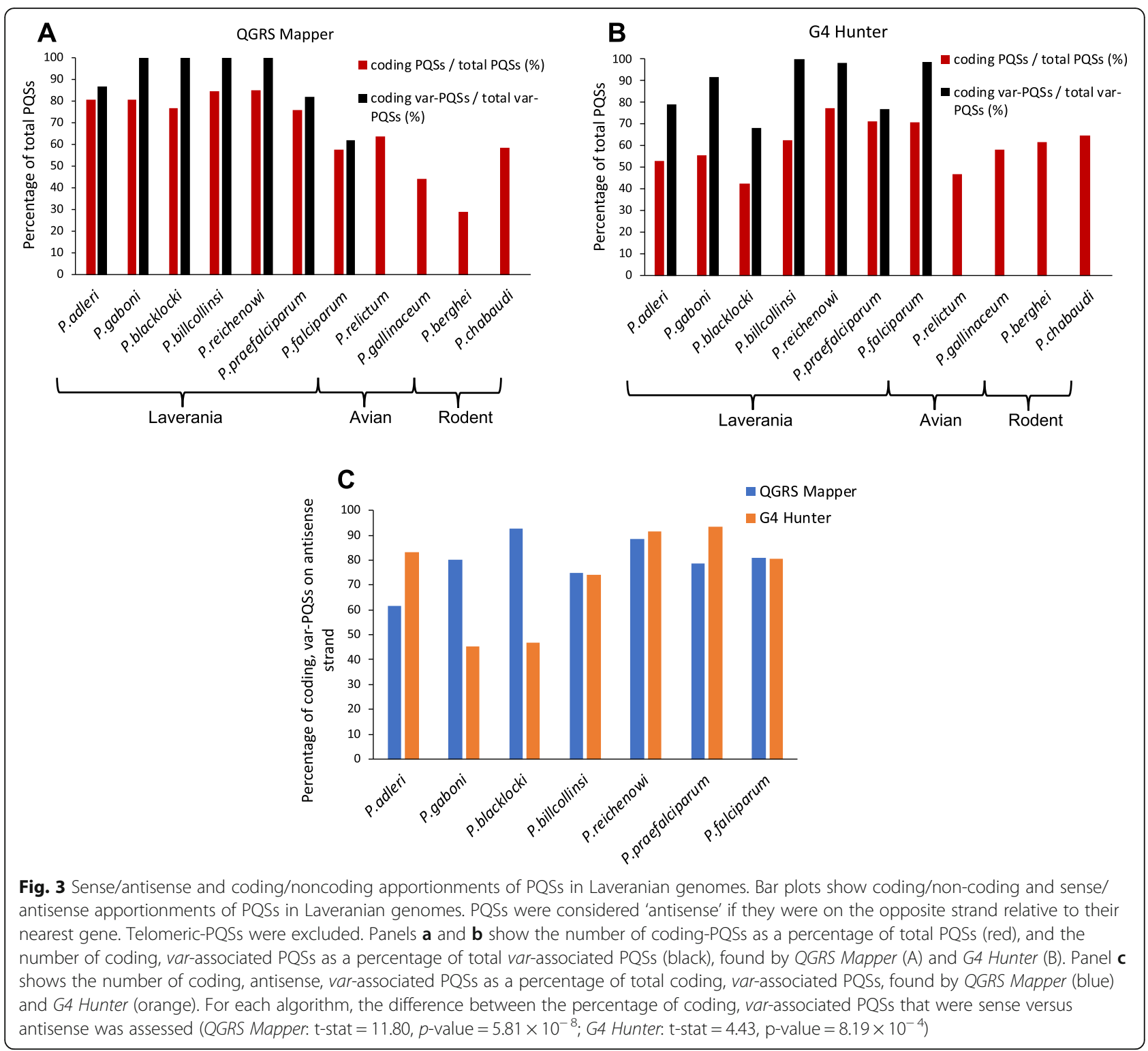

PQSs per var, as the majority of vars in the Clade A Laverania had one PQS, whereas the Clade B Laverania had a higher proportion of several-PQS var genes. $P$. blacklocki, which falls within Clade B but is the most evolutionary ancient in this clade, and thus 'closest' to Clade A, has a PQS distribution more closely resembling that seen in the Clade A var genes.

In their previous search for PQSs in P. falciparum and $P$. reichenowi, Stanton et al. reported that almost half of the var-associated PQSs in P. falciparum were located upstream of upsB-type var genes (an observation first made by Smargiassio et al. [17]), whereas this group was entirely absent in $P$. reichenowi, and almost all of the var-associated PQSs in P. reichenowi were coding [23]. 'UpsB' refers to a subgroup of the var gene family, which is frequently sub-classified according to similarity in the upstream ('ups') regions of the genes: upsB-type var genes are the largest subgroup in $P$. falciparum and are found immediately inside the telomeres of almost every chromosome, placing their upstream region directly adjacent to the subtelomeric repeats. We repeated this analysis for the newly published Laveranian genomes to determine whether the clustering of PQSs in upsB-var promoters was unique to P. falciparum. In our analysis, most var-PQSs in P. falciparum were indeed associated with upsB-type genes $(62$ and $44 \%$ of the total var-PQSs with QGRS Mapper and G4 Hunter, respectively), in concordance with the findings of Stanton et al. [23]. Other Laveranian species, including $P$. reichenowi, did have some upsB-var-PQSs, but they constituted less than $20 \%$ of the total (Additional file 4: Fig. S1), and were usually in coding regions rather than $5^{\prime}$ UTRs (Fig. 5). Finally, we noticed a prominent cluster of 


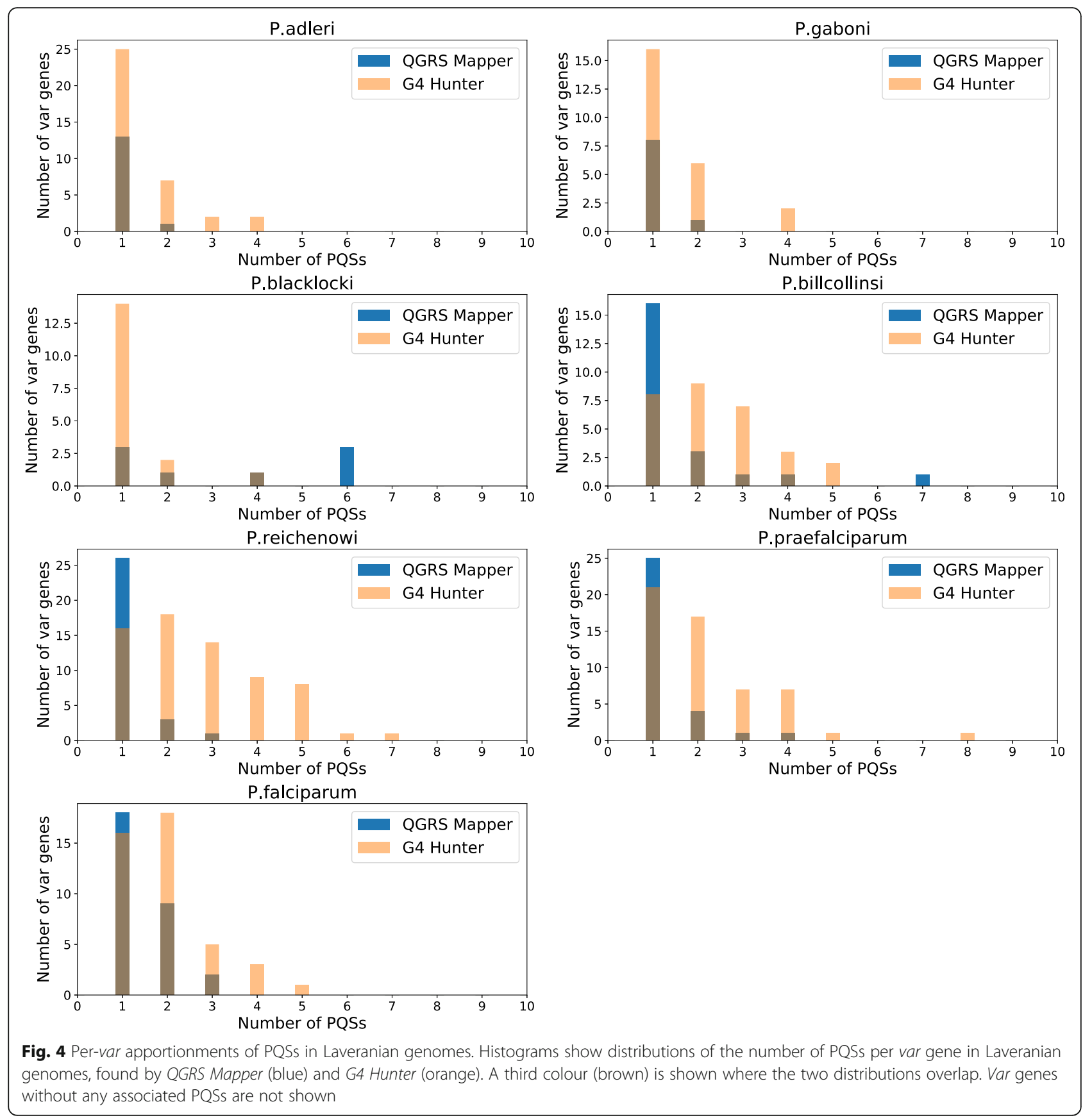

PQSs in most Clade-B species that was located $\sim 5 \mathrm{~kb}$ downstream of upsB-var start codons (Fig. 5). Thus, there is evidently a conserved bias in the location of PQSs within the coding sequences of these genes, but the noncoding, upstream PQSs are not conserved and appear to be unique to $P$. falciparum.

\section{G-quadruplex-forming motifs in Laveranian genomes are} not strongly associated with other multigene families Stanton et al. previously reported an association between PQSs and pir genes in P. berghei [23], suggesting that
PQSs could be co-distributed with variantly expressed virulence gene families in addition to var. The var gene family is uniquely present among Laverania, but other more ancient multigene families including pir, which may also have roles in virulence, have not been lost in these species: they still exist in varying numbers alongside the var genes. These other gene families are also highly variable in number: for example, some early Laverania have very few pirs, whereas $P$. falciparum has several hundred, but the early species instead have 30+ clags, whereas P. falciparum has only five [5]. We sought 


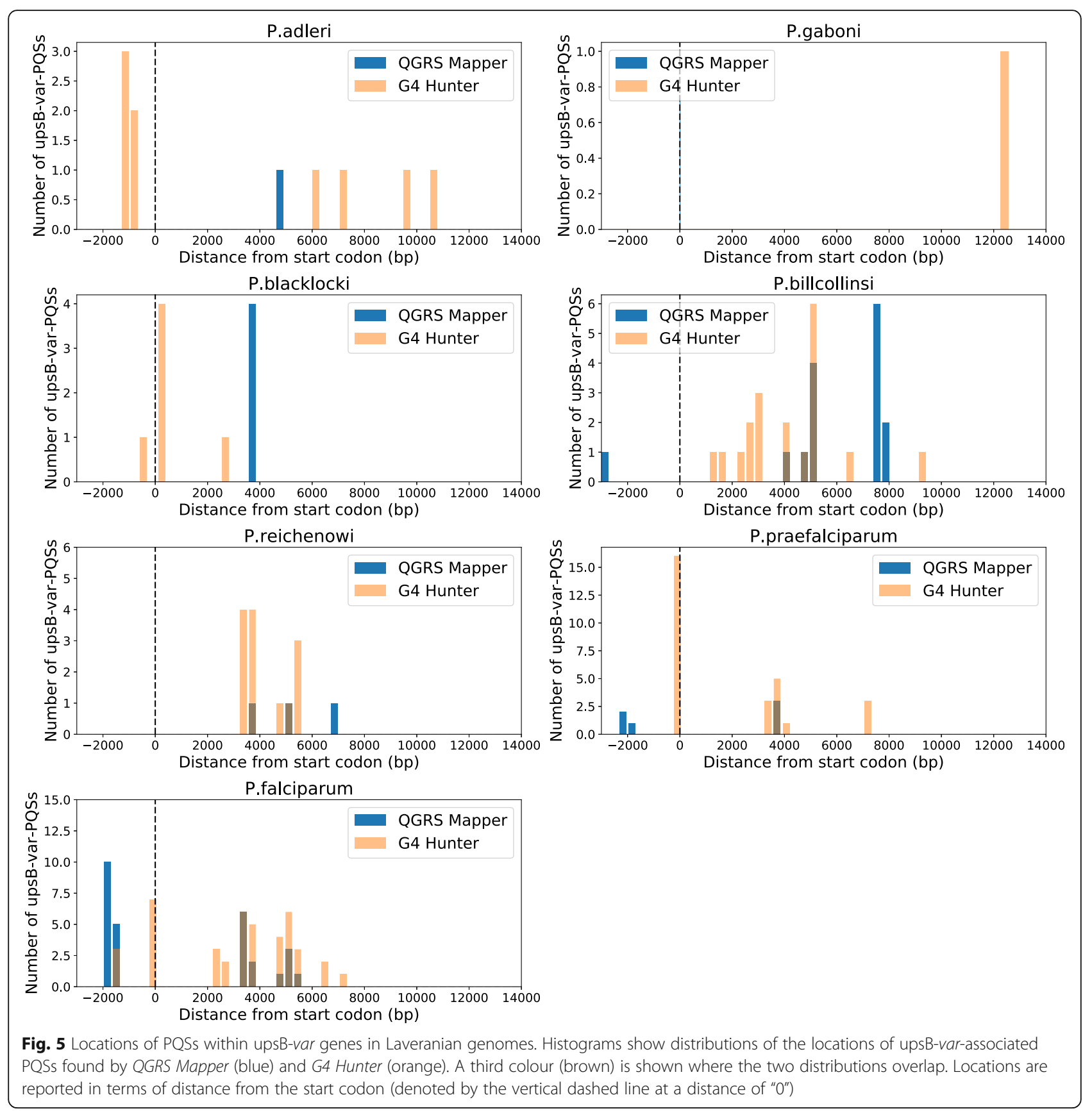

to establish whether any of these other families might be associated with G4s, and whether any evolutionary pattern could thus be discerned.

We searched the Laveranian genomes for codistributions of PQSs with other multigene families, including clag, PHIST, pir, rifin, and stevor. Surprisingly, we found very few PQSs in these gene families (Additional file 5: Fig. S2 and Additional file 6: Table S4). Neither algorithm predicted any PQSs in stevor genes. The clag family showed a weak association with PQSs in $P$. blacklocki, which has a large clag family (2 out of 35 clags, i.e., 6\%, had PQSs), and also in P. billcollinsi and P. reichenowi, which have smaller clag families (1 PQS amongst 7 and 6 clags respectively), while the PHIST family was moderately associated with PQSs in P. gaboni, P. adleri, $P$. billcollinsi, and $P$. praefalciparum (PQSs were found in 1 or 2 genes, representing $12.5,22,20$, and $8 \%$ of the total PHIST genes in each species, respectively). Additionally, some species had PQSs in a few pir genes and/or rifin genes, but the proportions were always small compared to the total sizes of the gene families. In all cases, the PQSs found in non-var multigene families were almost all found 
by G4 Hunter but not by QGRS Mapper, indicating that they are probably of non-canonical types. This is in contrast to the var-associated PQSs, many of which are canonical (Fig. 2).

\section{Numbers and distributions of G-quadruplex-forming motifs vary significantly in non-Laveranian Plasmodium genomes}

In addition to studying the Laveranian subgenus of Plasmodium, we analyzed the genomes of two more distantly related outgroups: species infecting rodents $(P$. berghei, $P$. chabaudi) and birds ( $P$. relictum, $P$. gallinaceum). These species do not have vars, but do have varying numbers of the other multigene families. We found significantly fewer PQSs in P. relictum and P. gallinaceum as compared to the Laverania (Welch's t-test, two-tailed, QGRS Mapper: $\quad \mathrm{t}$-stat $=3.92, \quad p$-value $=0.00718 ; \quad G 4$ Hunter: $\mathrm{t}$-stat $=4.35$, $\mathrm{p}$-value $=0.00355)$, while $P$. berghei and $P$. chabaudi had approximately the same number of PQSs as the Clade A Laverania (Fig. 2a). P. gallinaceum was unique in not having more PQSs found by G4 Hunter than QGRS Mapper.

By searching these genomes for associations between PQSs and multigene families (clag, PHIST, pir, rifin, stevor, reticulocyte-binding (Fig. 6)), we confirmed the codistribution of PQSs with pirs in P. berghei that was reported by Stanton et al. [23]: 37 of 217 pir genes had PQS(s) according to QGRS Mapper, i.e. 17\% of all pirs, and this constituted over $50 \%$ of the total genomic, nontelomeric PQSs. A significant, but markedly weaker, codistribution was also seen in P. chabaudi, with 11 of 208 pir genes having PQS(s) (Additional file 3: Table S3). Thus, this association may be common to pir genes of rodent malarias, but it is clearly weaker that that seen in var genes, and unlike the var genes it does not segregate with 'real' pir genes versus pir pseudogenes (pseudogenes are common in the $P$. berghei genome and many PQSs are found in these pseudogenes). Nevertheless, there is a contrast to the Laveranian genomes, where PQSs are rarely, if ever, in pirs: the two co-associate spatially simply due to the proximity between pir and

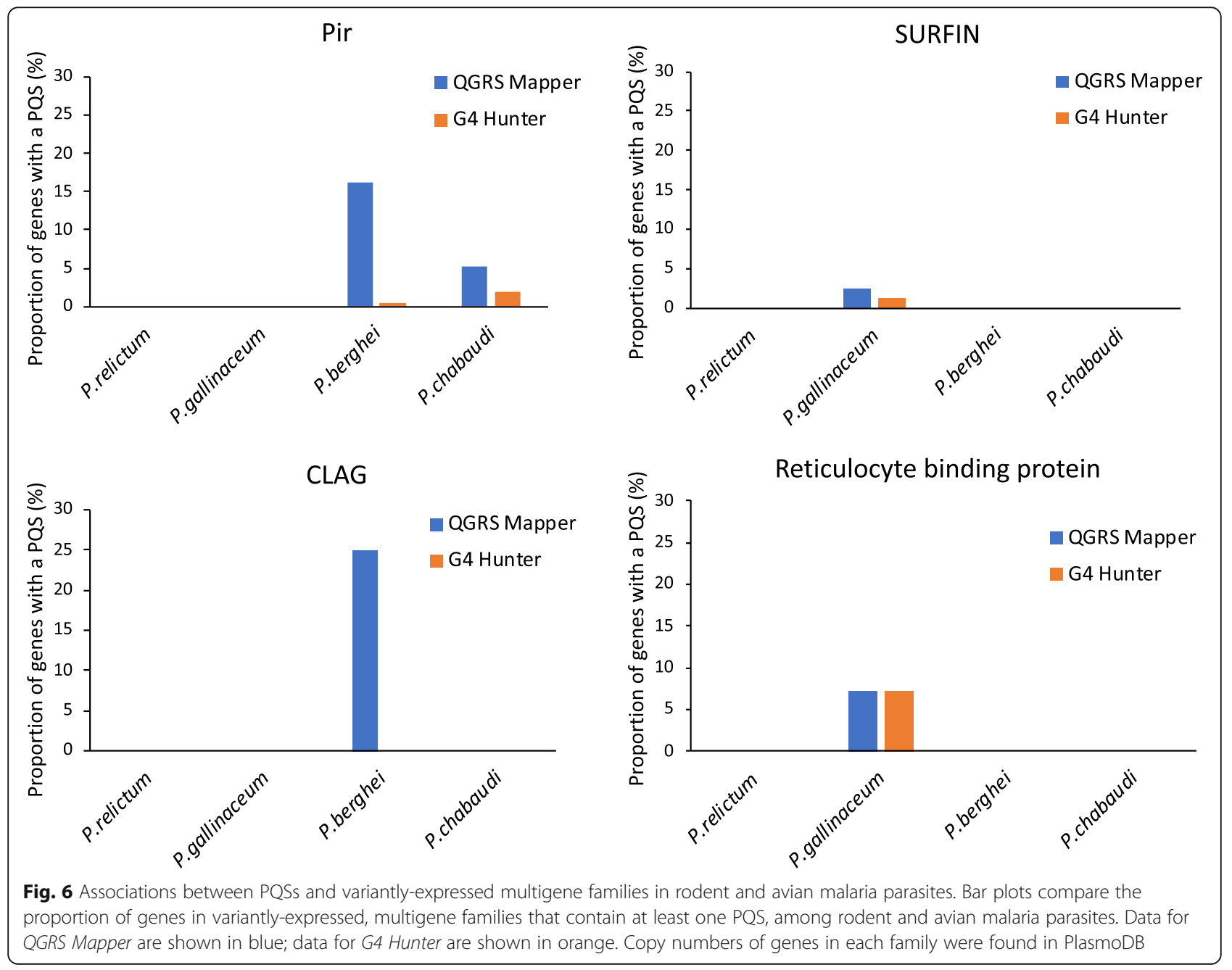


var genes. Notably, in both $P$. berghei and $P$. chabaudi, pir genes were exclusively associated with canonical PQSs as found by QGRS Mapper: the types of PQSs found by G4Hunter showed no association with pir genes. No other gene families harboured large numbers of PQSs in P. berghei, P. chabaudi, P. relictum, or P. gallinaceum (in $P$. berghei, one clag gene out of 4 harboured a PQS (Fig. 6), but given the small size of the gene family, this may be of limited relevance).

\section{Some non-var-G4s are conserved in Laveranian genomes}

Since the Laveranian genomes did not show strong associations between PQSs and multigene families other than var, we analyzed the degree to which non-var PQSs found anywhere else in these genomes might be conserved. We found that several PQS-gene associations were conserved (Additional file 7: Fig. S3): for example, all Laverania possessed at least one PQS in the genes encoding circumsporozoite protein, alpha/beta hydrolase, AP2 domain transcription factor(s), and SNARE proteins, whereas these associations were absent in the avian- and rodent-infecting Plasmodiums. Additionally, all Laverania except $P$. reichenowi possessed at least one PQS in the $40 \mathrm{~S}$ ribosomal protein $\mathrm{S} 2$-encoding gene, and all Laverania except $P$. blacklocki had at least one PQS in a PHIST gene. PQS-encoding genes were then clustered by GO-terms to seek any functional groups that might be particularly enriched in PQSs. Fig. 7 shows that the most prominent PQS-encoding genes were clearly the adhesin-encoding genes - i.e. primarily vars. A second group of helicaseencoding genes also contained conserved PQSs in all Laverania except P. falciparum (Fig. 7 and Additional file 8: Table S5).

\section{QGRS mapper, G4 hunter and G4-seq all give markedly different results on AT-rich Plasmodium genomes}

Finally, having conducted all the foregoing analyses with two different PQS-prediction algorithms, we compared their concordance. QGRS Mapper searches for sequences that match the general motif favorable for G4 formation, $G_{3} N_{(0-11)} G_{3} N_{(0-11)} G_{3} N_{(0-11)} G_{3}$, whereas $G 4$ Hunter does not require this 'canonical' form but

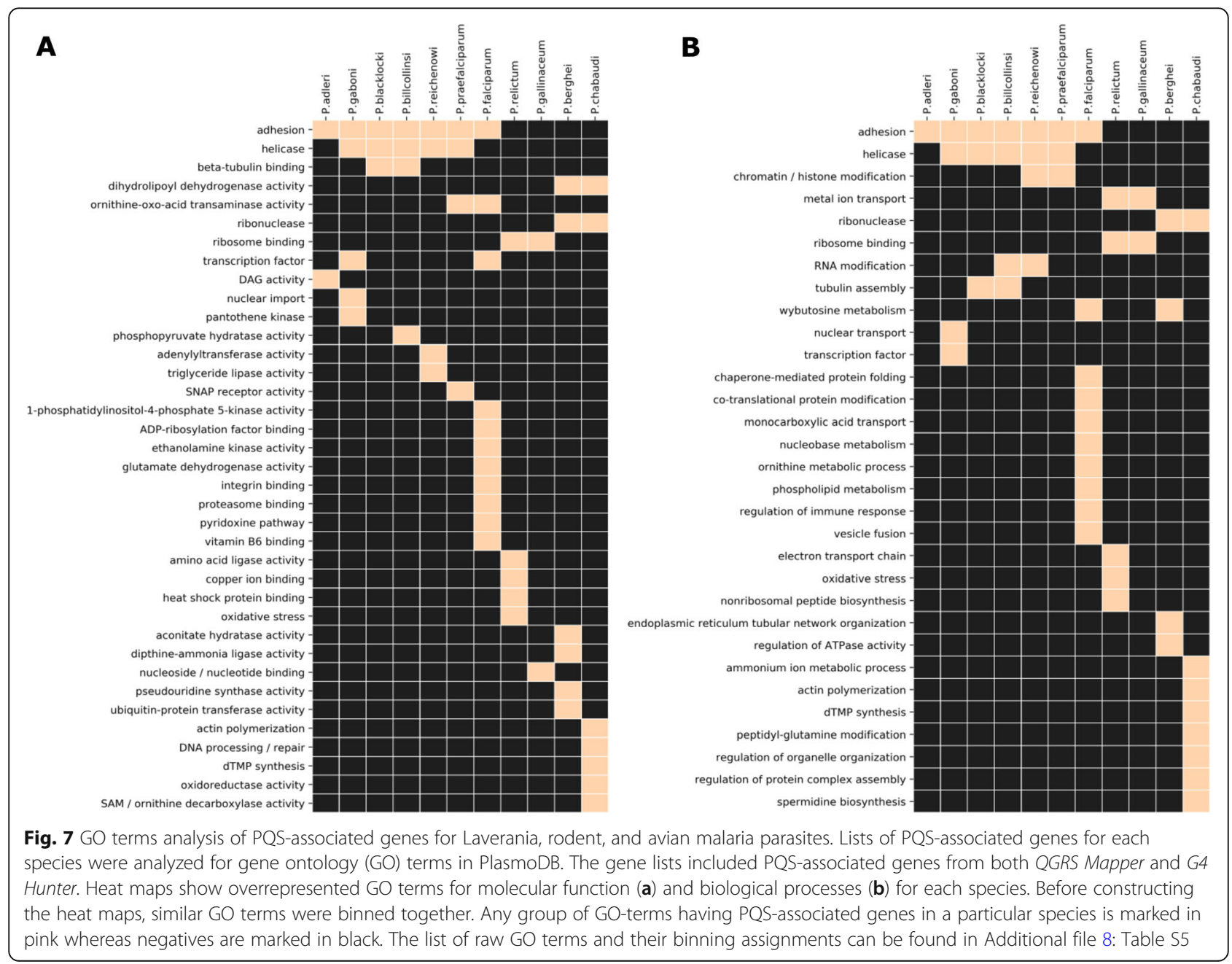


considers additional factors such as G-richness and Gskewness to calculate a score that reflects the propensity of a sequence to form a G4. G4 Hunter can therefore detect the "non-canonical" types of G4s that do not match the stereotypical pattern and would be overlooked by QGRS Mapper. By using both algorithms, we sought to characterize the proportions of canonical and noncanonical PQSs in Plasmodium genomes, and to compare the performance of the algorithms in general on AT-rich genomes. For all species except $P$. gallinaceum, G4 Hunter found more PQSs than QGRS Mapper, though this difference varied among species and did not track with any evolutionary pattern (Fig. 2a). Next, we investigated the degree of concordance between the two algorithms: for each species, we counted the number of PQS motifs, and also the number of unique PQScontaining genes, that were detected by both algorithms. We found a surprising lack of concordance (Fig. 8): only a minority of motifs were found in common. The percentage of common genes was somewhat greater, indicating that although both algorithms might find a PQS in the same gene, they usually did not find exactly the
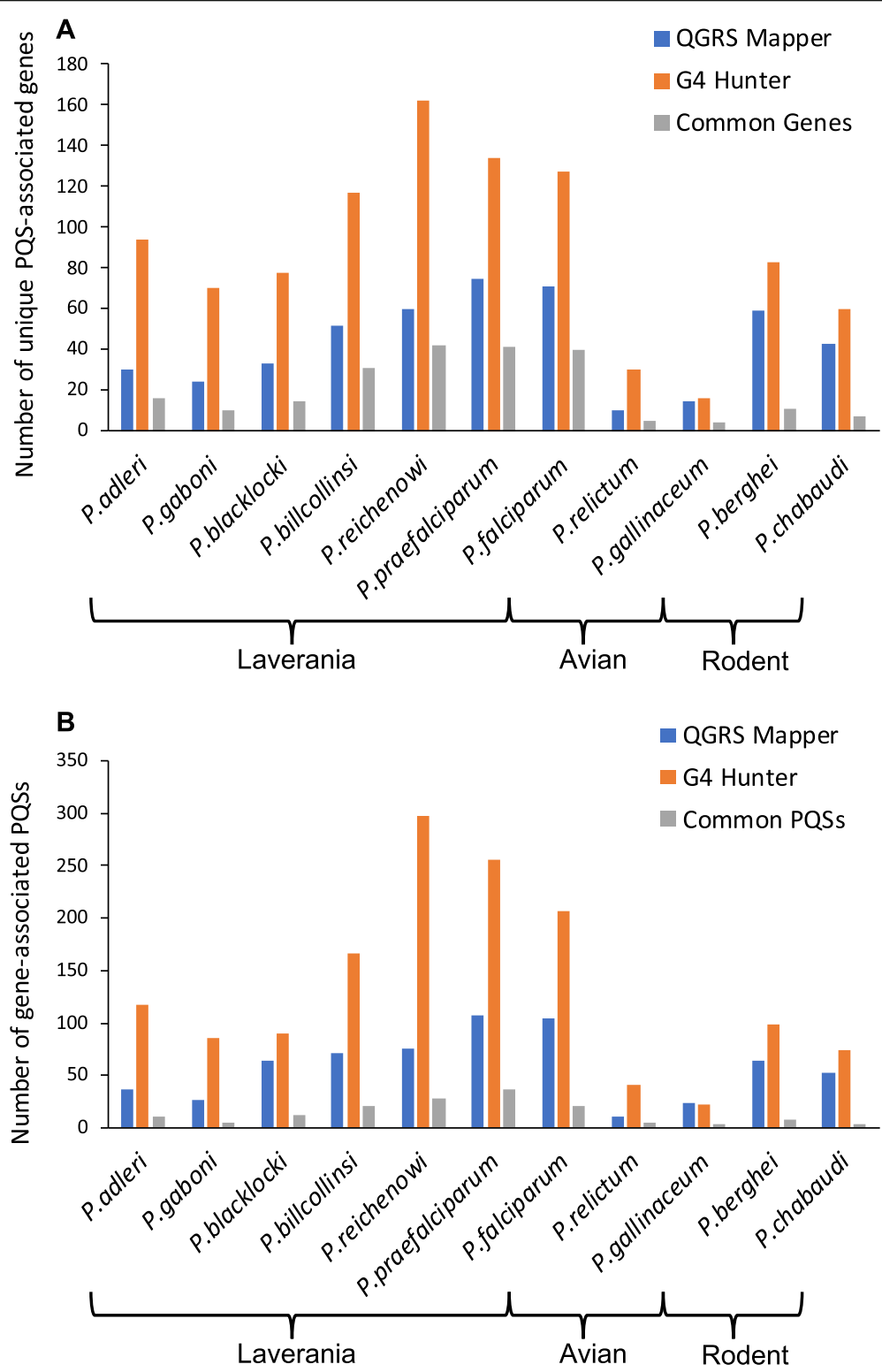

Fig. 8 Comparison of QGRS Mapper and G4 Hunter in their performance on AT-rich Plasmodium genomes. Bar plots compare the number of unique PQS-associated genes (a) and the number of gene-associated PQSs (b) found by QGRS Mapper (blue) and G4 Hunter (orange) for each Plasmodium species. Instances where both algorithms predicted at least one PQS in the same gene were considered "common genes" (A, grey). Instances where both algorithms predicted the same PQS were considered "common PQSs" (B, grey) 
same motif. Concordance also varied considerably between genomes, being relatively high in several Clade B Laverania and particularly low in the rodent and avian malaria genomes.

To test whether the lack of concordance was caused by setting an excessively high threshold for G4 Hunter, we re-analysed the $P$. falciparum genome at a lower threshold of 1.25 (this was initially set at 1.7 to reduce the risk of false positives and obtain a highly confident set of PQSs). Using a threshold of 1.25 increased the number of PQSs more than 10-fold, particularly in telomere repeats (Additional file 13: Table 8, and Additional file 9: Fig. S4) but it did not substantially increase concordance. Thus, even at a moderate threshold, G4 Hunter does not detect all the canonical motifs detected by QGRS Mapper; instead, the two algorithms appear to detect fundamentally different types of PQS. By experimentally testing the G4-folding capacity in three oligonucleotide sequences of new G4 Hunter PQSs, alongside two previously-published motifs found by QGRS Mapper, we showed that both types of PQS can indeed form G4s, at least in vitro (Additional file 10: Fig. S5).

We then compared the PQSs found by each algorithm with the locations of G4s that were experimentally observed in G4-seq on the P. falciparum genome - this being the only Plasmodium genome for which G4-seq has been performed [29] (Fig. 9a). G4-seq identified a total of 173 sites when the genome was sequenced in potassium-containing conditions, and twice as many, 326, in potassium-pluspyridostatin conditions (pyridostatin is a small molecule that stabilizes G4s and can thus increase the number of G4s that are detectable by sequencing [25]). It is clear that the overall number of sites detectable by G4-seq was greater than the number of unique (non-telomere) PQSs predicted by either in silico algorithm, but in fact $>80 \%$ of all sites found by G4-seq in the P. falciparum genome proved to be in telomeres, so only a small fraction of the predicted non-telomeric PQSs were actually found by sequencing. Fewer than one third of the total G4-seq sites had the potential to be predicted by QGRS Mapper using a loop size of $0-11$, because only $32.5 \%$ of the sequenced sites were canonical (defined by Marsico et al. as containing a $G_{3} N_{(0-12)} G_{3} N_{(0-12)} G_{3} N_{(0-12)} G_{3}$, motif) [29]. In fact, QGRS Mapper predicted 24 unique, non-telomererepeat sites that appeared in G4-seq (it did also predict a further 244 sites containing telomere repeats that were also picked up by G4-seq) (Fig. 9b,c). Excluding telomere repeats, however, only $22 \%$ of the total QGRS Mapper predictions for canonical G4s were verified by G4-seq. G4 Hunter, by contrast, could potentially have predicted any of the sequenced sites, but in fact predicted only 18 of them, so just $8.1 \%$ of G4 Hunter predictions were verified by G4seq (Fig. 9c, Additional file 11: Table S6).

\section{Discussion}

Our study shows that across all known Laveranian species, the co-distribution of G-quadruplex-forming motifs with var genes is strongly conserved. This was previously reported only in two closely-related species, $P$. falciparum and $P$. reichenowi, which have very similar numbers and arrangements of var genes [23]; we now show that it remains true across all seven species and at least five times as much evolutionary time, despite striking interspecies differences in the total numbers of var genes, the total numbers of PQSs and indeed the proportions of var-associated PQSs. Notably, Clade B Laverania had more PQSs overall in their genomes than Clade A species, suggesting that selection for these motifs may have increased rather than decreased across the evolutionary pathway. This did not appear to be exclusively due to strengthening selection for PQSs in var genes, because vars in Clade B were not universally more associated with PQSs than those in Clade A, nor did the species with the largest var gene families have the most numerous PQSs. However, we were unable to account for the differences by associating PQSs with any other variant gene family, or with any other gene or gene-class elsewhere in the genome. Overall, therefore, it appears that PQSs have been uniquely selected to associate with var genes across the Laverania. There are many possible reasons for this, but at present the main reason supported by experimental data is that G4s can promote beneficial diversity-generating recombination between var genes $[23,30]$.

We next examined in detail the distribution and location of PQSs in and around var genes. We observed that several var genes had more than one PQS - an unlikely event if these sparse motifs were randomly distributed across the genome, and one that emphasizes the apparent selection for PQSs in var genes. Furthermore, Clade B Laveranian species may be evolving towards having more PQSs per var, since PQS clusters were more common in var genes in Clade B than Clade A, and P. blacklocki, the most evolutionarily ancient species in Clade B, had a PQS distribution most closely resembling that seen in the Clade A vars. Secondly, the great majority of PQSs were in coding rather than UTR regions, with a distinct bias for the antisense strand. This could potentially allow the var genes to benefit from the recombination-promoting effects of G4s without suffering any interruption of codingstrand transcription, which could impede efficient production of PfEMP1. (We have previously showed that a stabilized coding-strand G4 can inhibit transcription of a rifin reporter gene [31], so the same could be true for G4encoding var genes.) Coding PQSs - at least amongst the upsB var genes that were analysed in Fig. 5 - occurred mostly around the middle of var gene sequences, within the second exon that encodes the variable extracellular 


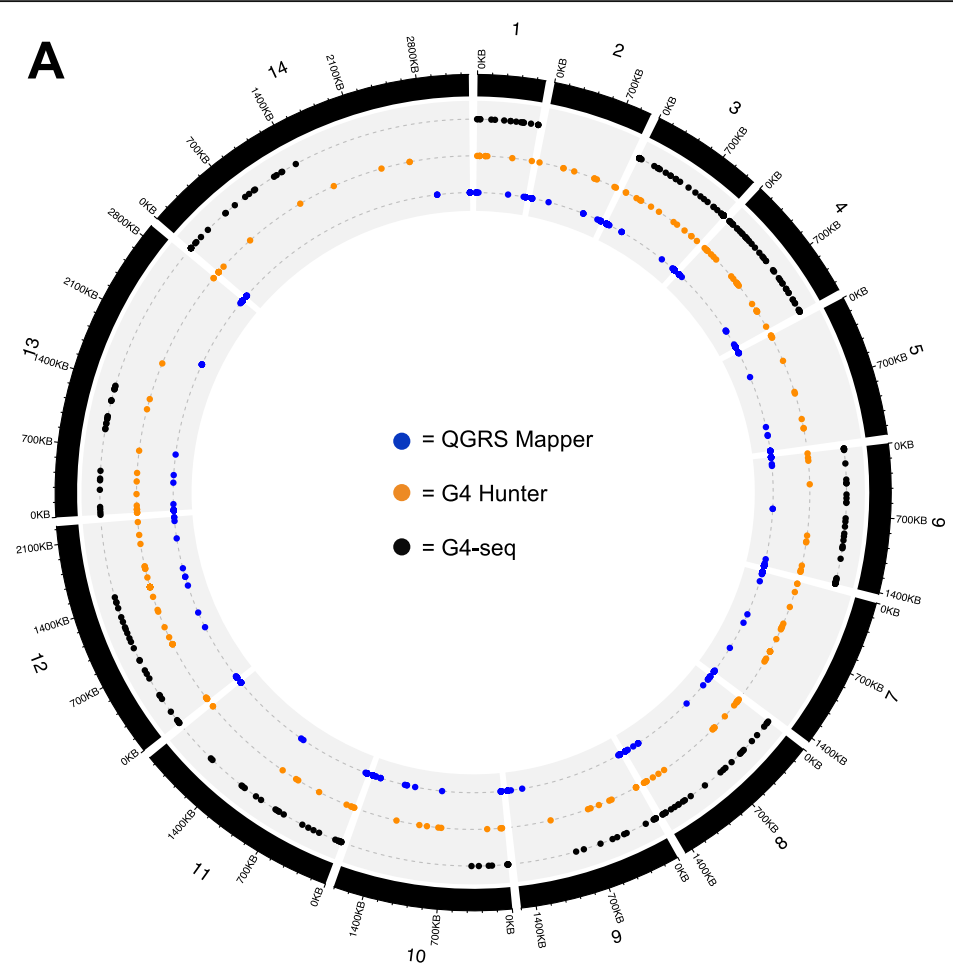

B

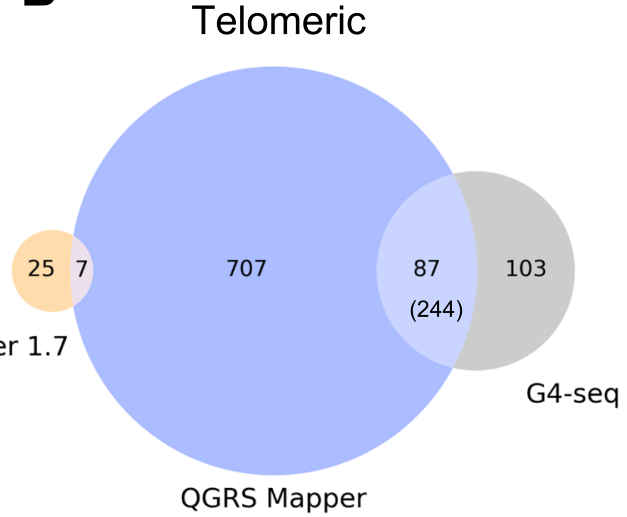

C

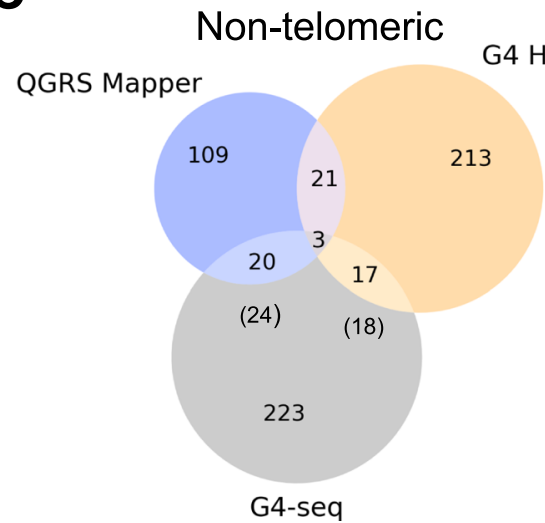

Fig. 9 Analysis of concordance among motifs predicted by QGRS Mapper, G4 Hunter and experimental G4-seq in the P. falciparum 3D7 genome. A circos plot (a) shows the distribution of PQSs across the 14 chromosomes in the P. falciparum 3D7 genome. Data from QGRS Mapper, G4 Hunter and experimental G4-seq are depicted by blue, orange and black circles, respectively. G4-seq yielded no data for chromosomes 2, 5 and 7 because these chromosomes were not covered in the original sequencing data (GEO accession GSM3003550). Note that telomeric PQSs with similar locations appear to cluster into a single data point, which visually minimizes the number of these PQSs (particularly in the case of QGRS Mapper, where many PQSs were detected within telomere repeats). Venn diagrams (b and $\mathbf{c}$ ) depict the overlaps between PQS sequences detected by QGRS Mapper, G4 Hunter (threshold=1.7), and experimental G4-seq. For G4-seq data, PQSs within 20 kb of chromosomal ends were considered telomeric. Due to the size of the G4-seq windows, it was possible for multiple PQSs to fall within the same window. Numbers in parentheses show the "full" count of PQSs detected within G4-seq windows, while numbers not in parentheses show the number of unique G4seq windows that contained at least one PQS

domain of PfEMP1. This would maximize the potential for DNA breaks resulting in beneficial domain-shuffling between var genes, which is known to occur in an organized and conservative manner in P. falciparum [15]. The conserved organization across the Clade B Laverania would again suggest that PQSs may function similarly in all these genomes. Thirdly, $P$. falciparum encoded an additional large set of PQSs upstream of upsB-type var genes, which appeared to be unique - i.e. not observed in other Laverania. It is intriguing to speculate that this may be a recent evolution in this species, perhaps selected to play a new role in transcriptional control of var genes (a known 
function of G4s upstream of oncogenes [19]). Alternatively, these G4s could buffer the transmission of chromatin states along DNA between the telomeres and the upsB genes [32].

Moving from the var genes to other variant gene families, we failed to find any strong association of PQSs with any other particular gene family in the Laverania. It was not the case, for example, that the strikingly expanded clag families in the more ancient species all harboured high numbers of PQSs. We did observe a few clag and PHIST genes that contained a (non-canonical) PQS but any biological significance of this remains unclear.

In two outgroups - avian and rodent malarias - we sought associations between PQSs and variant gene families that might hint at how the situation in the Laverania evolved, and furthermore might support the idea that G4s could play the same role in promoting recombination and diversification in any gene family that is under immune pressure. In $P$. berghei and $P$. chabaudi, PQSs were co-distributed with pir genes - albeit relatively weakly and only for canonical (QGRS Mapper) PQSs. There is indeed some evidence that pir genes encode variantly expressed virulence determinants that could theoretically benefit from mitotic diversification, analogous to that of the var genes [8,9]. Alternatively, it is possible that the G4s might act as transcriptional modulators, since the majority of pir-associated PQSs in $P$. berghei were non-coding, in contrast to the coding exon-2 bias of PQSs within var genes.

PQSs in the avian-malaria genomes were notably sparse, over and above what might be expected from their underlying genome bias. At 17.0 and $18.3 \% \mathrm{G} / \mathrm{C}, P$. gallinaceum and $P$. relictum are both lower than $P$. falciparum (19.3\%), but comparable to several other Laveranian genomes (17.6-18.6\%) which still harbour twice as many PQSs as either avian genome. The few PQSs that were found were not strongly associated with any gene family (clag, PHIST, pir, rifin or stevor) so, if the above theory is correct, it may be that no gene family in these avian parasites has evolved to diversify via G4-mediated recombination.

Besides the variant gene families, about half of the total PQSs in most Laveranian genomes were associated with other genes throughout the genome, so we assessed the conservation of these motifs as well. This proved to be limited: although certain genes such as the gene encoding circumsporozoite protein were clearly conserved complete with their PQS across the Laverania, only two functional groups of genes showed a highly conserved enrichment of PQSs and one of these was the vars (the other being helicase-encoding genes, see Fig. 7).

Finally, we compared QGRS Mapper and G4 Hunter in their performance on G/C-poor genomes, and found a surprising lack of concordance between the two algorithms. It was expected that G4 Hunter would find greater numbers of PQSs, due to its ability to accommodate non-canonical sequences, and the same has indeed been reported in other genomes [27]. However, G4 Hunter did not simply find all the canonical sequences and then some additional non-canonical ones. In some genomes, such as P. relictum, G4 Hunter did find $50 \%$ of the canonical sequences, but in $P$. chabaudi it found only $\sim 8 \%$. In fact, $P$. gallinaceum, which had extremely few PQSs overall, was unique in having the same numbers of PQSs found by both algorithms, but again, they were generally not the same motifs. This suggests that in some genomes, many or most of the canonical PQSs are not actually predicted to fold under stringent G4 Hunter conditions, perhaps due to long loop sizes or unfavourable G-richness or G-skewness in their sequence context. This is not unprecedented: the originators of $G 4$ Hunter reported that canonical PQSs in the myc promoter were not necessarily predicted by stringentlythresholded G4 Hunter [27]. Amongst Plasmodium, this phenomenon was much more marked in the outgroups than in the Laverania, perhaps suggesting that canonical G4s that actually do fold are positively selected in Laveranian genomes - consistent with the proposal that stable, folded G4s play a particular beneficial role in var gene organization.

Our analysis emphasizes that it is clearly important to check any in silico prediction experimentally. Very few Plasmodium sequences have been thus verified, and the two that have were selected a priori by QGRS Mapper. (Notably, neither of them - belonging to the rifin gene PF3D7_0700200 and the var upsB region [17, 31] - were found here by G4 Hunter: they presumably fall into the false negative category which is expected to be quite large at a threshold of 1.7.) Here, we experimentally tested three new G4 Hunter motifs, including two with the apparent potential to form only two-quartet rather than three-quartet G4s. All three were found to bind to the G4-specific dye thioflavin T, suggesting that PQSs found by G4 Hunter can fold as readily as those found by QGRS Mapper. The two algorithms appear to find fundamentally different types of motif, and should perhaps be viewed as complementary tools for G4 prediction.

Besides the biophysical characterization of individual sequences, genome-wide G4-seq has recently been conducted in P. falciparum [29] and this is an experimental rather than in silico method, albeit still performed in vitro on isolated DNA. In the human genome, G4-seq found more G4s than the original number predicted in silico, and many of those were non-canonical [25]. Similarly, more than two-thirds of the G4-seq sites found in the P. falciparum genome were 'non-canonical' [29] - 
an even greater proportion than in the human genome but they were predominantly not the sites predicted by G4 Hunter, with less than $10 \%$ overlap between the G4 Hunter predictions and the G4-seq sites. This raises several important points. Firstly, Plasmodium genomes probably harbour a high proportion of non-canonical G4s compared to the human genome, with potential implications for a) their stable folding in DNA versus RNA (2-quartet motifs are expected to be more stable in RNA), and b) for the proteins that might metabolize these structures. Secondly, the 'state of the art' algorithm G4 Hunter, at least when stringently applied, may not perform very accurately on the unusual PQS content of very $\mathrm{G} / \mathrm{C}$-poor genomes. Unexpectedly, the secondgeneration algorithm QGRS Mapper actually did better: $20 \%$ of its predicted sites were found to be folded in G4seq. Nevertheless, this is still a low proportion, and QGRS Mapper can predict only a minority of G4-seq sites in any genome where they are mostly noncanonical. Thirdly, caution is therefore advisable when using any in silico algorithm to predict G4s in Plasmodium genomes and experimental validation is especially important - in vitro and ideally in cellulo.

\section{Conclusion}

This research shows that G4-forming motifs are uniquely strongly associated with Plasmodium var genes, suggesting that Laveranian malaria parasites have evolved a particular role for G4s in the organization of var genes. Secondly, in the A/T-rich genomes of Plasmodium species, the choice of prediction algorithm may be particularly influential when studying G4s in these important protozoan pathogens.

\section{Methods}

\section{Organisms and genomic information}

Genome sequences of the genus Plasmodium were downloaded from PlasmoDB release 44 [33]. The following Plasmodium strains were used for analysis: $P$. adleri G01, P. gaboni G01, P. billcollinsi G01, P. blacklocki G01, $P$. reichenowi G01, $P$. praefalciparum G01, P. falciparum 3D7, P. relictum SGS1-like, P. gallinaceum 8A, P. berghei ANKA, and P. chabaudi chabaudi.

\section{In silico prediction of PQSs in Plasmodium genomes using} QGRS Mapper and G4 Hunter

PQSs were found throughout this work using the tools QGRS Mapper (version 1) [28] and G4 Hunter [27]. The parameters used for the two algorithms were: QGRS Mapper- regex: $G_{3} N_{(0-11)} G_{3} N_{(0-11)} G_{3} N_{(0-11)} G_{3}$, max length: 45, min G-group: 3, loop size: 0-11; G4 Hunterthreshold: 1.7 , window size: 25 , csv data: grouped. Bedrat et al. [27] used similar parameters while comparing the performance of the quadparser and G4 Hunter algorithms on the human mitochondrial genome: they found that these yielded low false positive rates while also predicting comparable numbers of PQSs. An additional analysis of the P. falciparum genome was conducted with a G4 Hunter-threshold of 1.25 instead of 1.7 .

For each species, each chromosome sequence was downloaded from PlasmoDB and analyzed using the QGRS Mapper and G4 Hunter web applications. QGRS Mapper does not simultaneously analyze both the sense and antisense strands, so reverse-complements of the chromosomes and contigs were generated and analyzed separately by this algorithm. For genomes with large numbers of contigs $(>5)$, the contig sequences were stripped of their FASTA headers, binned into a single .txt file, and analyzed together. Each PQS was then assigned to its associated contig by searching for the sequence in the list of contig sequences. Full lists of the sequence motifs found with each algorithm are tabulated in Additional files 12 and 13 (Tables S7 and S8).

\section{Parsing genomic coding sequences for PQS-associated gene annotations}

Files containing annotated coding sequences in FASTA format for each species were downloaded from PlasmoDB release 44. For each PQS location, the nearest gene ID and its annotation were found by parsing the list of coding sequences for the species. The distance between each PQS and the nearest gene was measured from the start of the PQS to the start or end of the coding sequence. If the PQS was found within a coding sequence, a distance of " 0 " was assigned. If the PQS location was downstream of the nearest gene, the distance was represented as a negative integer.

\section{Classification of telomeric and gene-associated PQSs}

PQSs predicted by QGRS Mapper and G4 Hunter were further classified as telomeric, gene-associated, and/or upsB-var gene-associated. Telomeric PQSs were identified as those with repeating 7-bp motifs (GGGTTT/CA), which are characteristic of telomeric sequences in Plasmodium spp. [34]. These telomeric PQSs were excluded from downstream analyses. The remaining, nontelomeric PQSs were classified as "gene-associated" if they were found within $2 \mathrm{~kb}$ of the beginning or end of a genomic coding sequence. An additional classification was made for PQSs that were associated with upsB-type var genes. Since the gene annotations in the v.44 assemblies did not specify particular var classes (e.g. ups-A, ups- $B$, ups- $C$ ), the assignments were made on the basis of gene location and orientation. upsB-type var genes were defined as those at the far ends of the chromosomes and transcribed inwards (with the $5^{\prime}$ UTR nearest the telomere). 


\section{Statistical analysis of the co-distribution of PQSs and multigene families}

Statistically significant associations between the locations of var/pir genes and PQSs were determined as per Stanton et. al. 2016 [23]. Briefly, the distance of every gene from its nearest PQS was calculated, measuring from the start or end of the PQS and scoring '0' for genes with a PQS within their coding sequence. A set of genes with random positions was then simulated as a comparator to test the null hypothesis of no association. The actual dataset and the simulated datasets were analyzed to determine the mean and median distance from PQSs, with the actual distance being compared to the null situation. The statistical significance of each mean difference was assessed using Welch's t-test (2-tailed), with significance set at $p \leq 0.05$, or for each median distance, by calculating a 95\% confidence interval around the median.

For genome assemblies that contained contigs, these were treated as discrete chromosomes. Additionally, vars/pirs on chromosomes and contigs that did not contain any PQSs had to be excluded, since the 'distance to nearest PQS' did not exist. In P. berghei and P. chabaudi, which each encode an isolated group of pir genes more than $1 \mathrm{Mb}$ from the nearest PQS, these marked outliers were excluded.

\section{Gene ontology (GO) term enrichment analysis of PQS- associated genes}

For each species, a list of PQS-associated genes was compiled from the QGRS Mapper and G4 Hunter datasets. The lists were analyzed for enriched GO terms using the Gene Ontology Enrichment tool in PlasmoDB [33], with parameters: ontology: molecular function and biological process, evidence: computed and curated, limit to GO Slim terms: No, p-value cutoff: 0.05. Similar GO terms from the resulting datasets were binned together (Additional file 8: Table S5) and visualized with heatmaps, which were constructed using the Seaborn library in Jupyter Notebook.

\section{Comparing QGRS Mapper, G4 Hunter, and G4-seq in their analysis of AT-rich Plasmodium genomes}

QGRS Mapper and G4 Hunter were compared by searching for instances where both algorithms predicted the same PQS, or where the PQS of one algorithm was a "substring" of a sequence predicted by the other algorithm. The in silico predictions were compared to experimental G4-seq data [29] by searching for instances where PQS start/end locations overlapped with G4-seq window locations. A circos plot of PQS locations was constructed using the shinyCircos library in R Studio, and Venn diagrams were constructed using the venn3 library in Python 3 [35].

\section{Statistical testing for comparison of means and distributions}

Comparisons between Clade A Laverania, Clade B Laverania, rodent malaria parasites and avian malaria parasites often involved groups with unequal sample sizes and variances. In these cases, the two-sided Welch's t-test was chosen, since it makes no assumptions regarding equality of sample sizes and variances. Descriptive statistics were calculated using Microsoft Excel. Hypothesis testing was performed using the Scipy library in Python 3. All statistical tests were interpreted at a significance level of 0.05 .

\section{G4 folding assay}

PQS oligonucleotides were tested for their ability to fold into G4s using the G4-specific fluorescent dye thioflavin $\mathrm{T}$ [36]. Each oligonucleotide was tested alongside a sequence-scrambled version. $20 \mu \mathrm{M}$ oligonucleotides were heated to $90{ }^{\circ} \mathrm{C}$ for 5 mins and buffer was added at $100 \mu \mathrm{M}$ Tris pH 7.8, $100 \mu \mathrm{M} \mathrm{KCl}$. Cooling from $90^{\circ} \mathrm{C}$ to room temperature was then controlled at a rate of $5^{\circ} \mathrm{C} / 5$ mins. $80 \mu \mathrm{M}$ thioflavin $\mathrm{T}$ (Sigma) was added and incubated at room temperature for 5 mins. $40 \mu \mathrm{l}$ of each sample was transferred in triplicate to a 96-well, black, Uclear plate (Greiner) and fluorescence was measured (Ex. 420 nm, Em. $480 \mathrm{~nm}$ ) using a FLUOstar Omega plate reader (BMG Labtech).

\section{Custom code for data collection and analysis}

All parsing was performed using Python 3.6.5. Additional data analysis, visualization, and hypothesis testing were performed using Jupyter Notebook. Code is available at https://github.com/hgage/G4s-across-Plasmodium-evolution.

\section{Supplementary information}

Supplementary information accompanies this paper at https://doi.org/10. $1186 /$ s12864-020-6625-X.

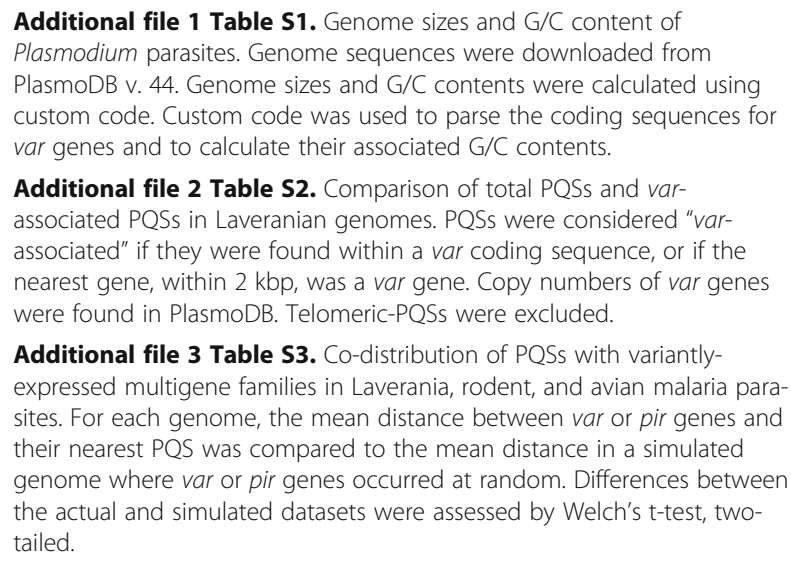

Additional file $\mathbf{3}$ Table S3. Co-distribution of PQSs with variantlyexpressed multigene families in Laverania, rodent, and avian malaria parasites. For each genome, the mean distance between var or pir genes and their nearest PQS was compared to the mean distance in a simulated genome where var or pir genes occurred at random. Differences between the actual and simulated datasets were assessed by Welch's t-test, twotailed. 
Additional file 4 Fig. S1. PQSs associated with upsB-type var genes in Laveranian genomes. Bar plots show the number of upsB-var-associated PQSs as a percentage of total var-associated PQSs, found by QGRS Mapper (blue), and G4 Hunter (orange), in Laveranian genomes. PQSs were considered "upsB-var-associated" if they were found within the coding region of a upsB-type var gene, or if the nearest gene, within $2 \mathrm{kbp}$, was a upsB-type var gene.

Additional file $\mathbf{5}$ Fig. S2. Associations between PQSs and variantlyexpressed multigene families besides var in Laveranian genomes. Bar plots compare the number (A) and proportion (B) of genes in non-var variantly-expressed multigene families that contain at least one PQS, among Laveranian species. Data for QGRS Mapper is shown in blue; data for G4 Hunter is shown in orange. Copy numbers of genes in each family were found in PlasmoDB.

Additional file 6 Table S4. Associations between PQSs and variantlyexpressed multigene families besides var in Laveranian genomes. Numbers and proportions of genes in non-var variantly-expressed multigene families that contain at least one PQS, in Laveranian species. Copy numbers of genes in each family were found in PlasmoDB.

Additional file 7 Fig. S3. Conserved PQS-associated genes in Plasmodium species. For each species, we compiled a list PQS-associated gene IDs (found by either QGRS Mapper or G4 Hunter), as well as their gene annotations. The table lists PQS-associated gene annotations that were common among certain groups of Plasmodium species.

Additional file 8 Table S5. GO terms analysis of PQS-associated genes for Laverania, rodent, and avian malaria parasites. Lists of PQS-associated genes for each species were analyzed for gene ontology (GO) terms in PlasmoDB. The table shows overrepresented GO terms for molecular function and biological processes for each species, as well as binning assignments that were used for the construction of the heat maps in Fig. 7.

Additional file $\mathbf{9}$ Fig. S4. Analysis of concordance among motifs predicted by QGRS Mapper, low-threshold G4 Hunter and experimental G4 seq in the P. falciparum 3D7 genome. Venn diagrams show overlaps between PQS sequences detected by QGRS Mapper, G4 Hunter and experimental G4-seq. The concordance analysis was performed with data from G4 Hunter at two different thresholds: the more stringent threshold of 1.7 was used in Fig. $9 \mathrm{~B}$ and $\mathrm{C}$, whereas a threshold of 1.25 is depicted here. For G4-seq data, For G4-seq data, PQSs within 20 kb of chromosomal ends were considered telomeric. Due to the size of the G4-seq windows, it was possible for multiple PQSs to fall within the same window. Numbers in parentheses show the "full" count of PQSs detected within G4-seq windows, while numbers not in parentheses show the number of unique G4-seq windows that contained at least one PQS.

Additional file $\mathbf{1 0}$ Fig. S5. Analysis of G4-folding capacity in motifs found by G4 Hunter and QGRS Mapper. Bar plots (A, B) show fluorescent emission of the G4-specific dye thioflavin T in the presence of PQS oligonucleotides, scrambled-sequence controls, and a duplex of $A / T$ sequence that does not form a G4 (representing background emission in the presence of DNA). 'Relative fluorescence' is relative to that of thioflavin T alone (no DNA). (A) shows two canonical PQSs from $P$. falciparum that were previously characterised as G4-folding via several biophysical assays $[31,37]$. (B) shows three new PQSs predicted bv G4 Hunter in several Laveranian genomes. Data are the mean of $n=4$ experiments conducted in technical triplicate; error bars are SD; ****, $P<0.0001$. Table (C) shows the oligonucleotide sequences used.

Additional file 11 Table S6. Comparison of QGRS Mapper and G4 Hunter with experimental G4-seq data. The in silico predictions by QGRS Mapper and G4 Hunter were compared to experimental G4-seq data [29] by searching for instances where PQS start/end locations overlapped with G4-seq window locations. The table shows PQS sites and indicates whether they were found within a G4-seq window.

Additional file 12 Table S7. Putative G-quadruplex-forming sequences in the genomes of Plasmodium parasites, found by QGRS Mapper. Genomes were downloaded from PlasmoDB, v. 44, and searched using QGRS Mapper with the parameters: regex: $G_{3} N_{(0-11)} G_{3} N_{(0-11)} G_{3} N_{(0-11)} G_{3}$, max length: 45, min G-group: 3, loop size: 0-11. "RC_Location Calculated" refers to the location of PQSs on the antisense strand, relative to the $5^{\prime}$ end of the sense strand. The "Strand (cf gene or nearest gene)" category was denoted as antisense if the PQS was on the opposite strand of its nearest gene, or sense if the PQS was on the same strand as its nearest gene. The "distance from nearest gene" was positive if the PQS was upstream of the nearest gene, negative if the PQS was downstream of the nearest gene, or equal to 0 if the PQS was in the coding sequence of the nearest gene.

Additional file 13 Table S8. Putative G-quadruplex-forming sequences in the genomes of Plasmodium parasites, found by G4 Hunter. Genomes were downloaded from PlasmoDB, v. 44, and searched using G4 Hunter with the parameters: threshold: 1.7 , window size: 25 , csv data: grouped. An additional search of the $P$. falciparum genome was also conducted at a lower threshold of 1.25. As per the G4 Hunter algorithm, PQSs on the reverse complement sequence had negative scores, and all locations were reported relative to the 5 ' end of the sense strand. The "Strand (cf gene or nearest gene)" category was denoted as antisense if the PQS was on the opposite strand of its nearest gene, or sense if the PQS was on the same strand as its nearest gene. The "distance from nearest gene" was positive if the PQS was upstream of the nearest gene, negative if the $P Q S$ was downstream of the nearest gene, or equal to 0 if the PQS was in the coding sequence of the nearest gene.

\section{Abbreviations}

G4: G-quadruplex; GO: Gene Ontology; PfEMP1: Plasmodium falciparum erythrocyte membrane protein 1; pir: Plasmodium interspersed repeat; PQS: Putative quadruplex sequence; ups: Upstream sequence

\section{Acknowledgements}

We are grateful to Francis Totanes and Franck Dumetz for helpful discussions, Anders Jensen for help with G4 folding assays, and Holly Craven for critical reading of the manuscript.

\section{Authors' contributions}

HG mapped PQSs in Plasmodium genomes, analysed data, wrote and deployed algorithms, and wrote the manuscript. CJM designed the study and wrote the manuscript. Both authors read and approved the final manuscript.

\section{Funding}

The work was supported by UK Research Council grant MR/P010873 to C.J.M. The funder had no role in design of the study, analysis or interpretation of data, or in writing the manuscript.

\section{Availability of data and materials}

The original datasets supporting the conclusions of this article are available in the published literature [29] or are included within the article and its additional files. The code used in the analysis is available at https://github. com/hgage/G4s-across-Plasmodium-evolution.

\section{Ethics approval and consent to participate}

Not applicable.

\section{Consent for publication}

Not applicable.

\section{Competing interests}

The authors declare that they have no competing interests.

Received: 13 November 2019 Accepted: 26 February 2020 Published online: 17 March 2020

\section{References}

1. WHO: World Malaria Report 2018. 2018.

2. Smith JD, Chitnis CE, Craig AG, Roberts DJ, Hudson-Taylor DE, Peterson DS, Pinches R, Newbold Cl, Miller LH. Switches in expression of Plasmodium falciparum var genes correlate with changes in antigenic and cytoadherent phenotypes of infected erythrocytes. Cell. 1995;82(1):101-10.

3. Su XZ, Heatwole VM, Wertheimer SP, Guinet F, Herrfeldt JA, Peterson DS, Ravetch JA, Wellems TE. The large diverse gene family var encodes proteins involved in cytoadherence and antigenic variation of Plasmodium falciparum-infected erythrocytes. Cell. 1995;82(1):89-100. 
4. Baruch DI, Pasloske BL, Singh HB, Bi X, Ma XC, Feldman M, Taraschi TF, Howard RJ. Cloning the P. falciparum gene encoding PfEMP1, a malarial variant antigen and adherence receptor on the surface of parasitized human erythrocytes. Cell. 1995;82(1):77-87.

5. Otto TD, Gilabert A, Crellen T, Bohme U, Arnathau C, Sanders M, Oyola SO,

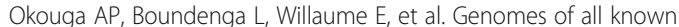
members of a Plasmodium subgenus reveal paths to virulent human malaria. Nat Microbiol. 2018;3(6):687-97.

6. Al-Khedery B, Barnwell JW, Galinski MR. Antigenic variation in malaria: a 3' genomic alteration associated with the expression of a P. knowlesi variant antigen. Mol Cell. 1999;3(2):131-41.

7. Brown KN, Brown IN. Immunity to malaria: antigenic variation in chronic infections of Plasmodium knowlesi. Nature. 1965;208(5017):1286-8.

8. Yam XY, Brugat T, Siau A, Lawton J, Wong DS, Farah A, Twang JS, Gao X, Langhorne J, Preiser PR. Characterization of the Plasmodium interspersed repeats (PIR) proteins of Plasmodium chabaudi indicates functional diversity. Sci Rep. 2016;6:23449.

9. Janssen CS, Barrett MP, Turner CM, Phillips RS. A large gene family for putative variant antigens shared by human and rodent malaria parasites. Proc Biol Sci. 2002;269(1489):431-6.

10. Bohme U, Otto TD, Cotton JA, Steinbiss S, Sanders M, Oyola SO, Nicot A, Gandon S, Patra KP, Herd C, et al. Complete avian malaria parasite genomes reveal features associated with lineage-specific evolution in birds and mammals. Genome Res. 2018;28(4):547-60.

11. Crabbe L, Verdun RE, Haggblom Cl, Karlseder J. Defective telomere lagging strand synthesis in cells lacking WRN helicase activity. Science. 2004; 306(5703):1951-3.

12. Scherf A, Hernandez-Rivas R, Buffet P, Bottius E, Benatar C, Pouvelle B, Gysin J, Lanzer M. Antigenic variation in malaria: in situ switching, relaxed and mutually exclusive transcription of var genes during intra-erythrocytic development in Plasmodium falciparum. EMBO J. 1998;17(18):5418-26.

13. Lapp SA, Korir-Morrison C, Jiang J, Bai Y, Corredor V, Galinski MR. Spleendependent regulation of antigenic variation in malaria parasites: Plasmodium knowlesi SICAvar expression profiles in splenic and asplenic hosts. PLoS One. 2013;8(10):e78014.

14. Cunningham D, Lawton J, Jarra W, Preiser $P$, Langhorne J. The pir multigene family of Plasmodium: antigenic variation and beyond. Mol Biochem Parasitol. 2010;170(2):65-73.

15. Claessens A, Hamilton WL, Kekre M, Otto TD, Faizullabhoy A, Rayner JC, Kwiatkowski D. Generation of antigenic diversity in Plasmodium falciparum by structured rearrangement of Var genes during mitosis. PLoS Genet. 2014; 10(12):e1004812

16. Freitas-Junior LH, Bottius E, Pirrit LA, Deitsch KW, Scheidig C, Guinet F, Nehrbass U, Wellems TE, Scherf A. Frequent ectopic recombination of virulence factor genes in telomeric chromosome clusters of P. falciparum. Nature. 2000;407(6807):1018-22.

17. Smargiasso N, Gabelica V, Damblon C, Rosu F, De Pauw E, Teulade-Fichou MP, Rowe JA, Claessens A. Putative DNA G-quadruplex formation within the promoters of Plasmodium falciparum var genes. BMC Genomics. 2009;10: 362.

18. Biffi G, Tannahill D, McCafferty J, Balasubramanian S. Quantitative visualization of DNA G-quadruplex structures in human cells. Nat Chem. 2013:5(3):182-6.

19. Siddiqui-Jain A, Grand CL, Bearss DJ, Hurley LH. Direct evidence for a Gquadruplex in a promoter region and its targeting with a small molecule to repress C-MYC transcription. Proc Natl Acad Sci U S A. 2002;99(18):11593-8.

20. Lopes J, Piazza A, Bermejo R, Kriegsman B, Colosio A, Teulade-Fichou MP, Foiani M, Nicolas A. G-quadruplex-induced instability during leading-strand replication. EMBO J. 2011;30(19):4033-46.

21. Paeschke K, Simonsson T, Postberg J, Rhodes D, Lipps HJ. Telomere end binding proteins control the formation of G-quadruplex DNA structures in vivo. Nat Struct Mol Biol. 2005;12(10):847-54.

22. Videvall E. Plasmodium parasites of birds have the most AT-rich genes of eukaryotes. Microb Genom. 2018;4(2):1-9.

23. Stanton A, Harris LM, Graham G, Merrick CJ. Recombination events among virulence genes in malaria parasites are associated with G-quadruplexforming DNA motifs. BMC Genomics. 2016;17(1):859.

24. Claessens A, Harris LM, Stanojcic S, Chappell L, Stanton A, Kuk N, VenezianoBroccia P, Sterkers Y, Rayner JC, Merrick CJ. RecQ helicases in the malaria parasite Plasmodium falciparum affect genome stability, gene expression patterns and DNA replication dynamics. PLoS Genet. 2018;14(7):e1007490.
25. Chambers VS, Marsico G, Boutell JM, Di Antonio M, Smith GP, Balasubramanian S. High-throughput sequencing of DNA G-quadruplex structures in the human genome. Nat Biotechnol. 2015;33(8):877-81.

26. Kwok CK, Merrick CJ. G-Quadruplexes: prediction, characterization, and biological application. Trends Biotechnol. 2017;35(10):997-1013.

27. Bedrat A, Lacroix L, Mergny JL. Re-evaluation of G-quadruplex propensity with G4Hunter. Nucleic Acids Res. 2016;44(4):1746-59.

28. Kikin O, D'Antonio L, Bagga PS. QGRS Mapper: a web-based server for predicting G-quadruplexes in nucleotide sequences. Nucleic Acids Res. 2006;34(Web Server issue):W676-82.

29. Marsico G, Chambers VS, Sahakyan AB, McCauley P, Boutell JM, Antonio MD, Balasubramanian S. Whole genome experimental maps of DNA Gquadruplexes in multiple species. Nucleic Acids Res. 2019;47(8):3862-74.

30. Harris LM, Merrick CJ. G-quadruplexes in pathogens: a common route to virulence control? PLoS Pathog. 2015:11(2):e1004562.

31. Harris LM, Monsell KR, Noulin F, Famodimu MT, Smargiasso N, Damblon C, Horrocks P, Merrick CJ. G-Quadruplex DNA Motifs in the Malaria Parasite Plasmodium falciparum and Their Potential as Novel Antimalarial Drug Targets. Antimicrob Agents Chemother. 2018;62(3).

32. Xia Y, Zheng KW, He YD, Liu HH, Wen CJ, Hao YH, Tan Z. Transmission of dynamic supercoiling in linear and multi-way branched DNAs and its regulation revealed by a fluorescent G-quadruplex torsion sensor. Nucleic Acids Res. 2018;46(14):7418-24

33. Aurrecoechea C, Brestelli J, Brunk BP, Dommer J, Fischer S, Gajria B, Gao X, Gingle A, Grant G, Harb OS, et al. PlasmoDB: a functional genomic database for malaria parasites. Nucleic Acids Res. 2009:37(Database issue):D539-43.

34. Ponzi M, Pace T, Dore E, Frontali C. Identification of a telomeric DNA sequence in Plasmodium berghei. EMBO J. 1985:4(11):2991-5.

35. Yu Y, Ouyang $Y$, Yao W. Shinycircos: an R/shiny application for interactive creation of Circos plot. Bioinformatics. 2018;34(7):1229-31.

36. Renaud de la Faverie A, Guedin A, Bedrat A, Yatsunyk LA, Mergny JL. Thioflavin $T$ as a fluorescence light-up probe for G4 formation. Nucleic Acids Res. 2014:42(8):e65.

37. De Cian A, Grellier P, Mouray E, Depoix D, Bertrand H, Monchaud D, Teulade-Fichou MP, Mergny JL, Alberti P. Plasmodium telomeric sequences: structure, stability and quadruplex targeting by small compounds. Chembiochem. 2008:9(16):2730-9.

\section{Publisher's Note}

Springer Nature remains neutral with regard to jurisdictional claims in published maps and institutional affiliations.

Ready to submit your research? Choose BMC and benefit from:

- fast, convenient online submission

- thorough peer review by experienced researchers in your field

- rapid publication on acceptance

- support for research data, including large and complex data types

- gold Open Access which fosters wider collaboration and increased citations

- maximum visibility for your research: over $100 \mathrm{M}$ website views per year

At $\mathrm{BMC}$, research is always in progress.

Learn more biomedcentral.com/submission 